\title{
DESIGNING FOR QUANTUM COMPUTING
}

\author{
Andrea Alessandro Gasparini \\ Department of Informatics \& University of Oslo Library, \\ University of Oslo, P. Boks 1080, 0316 Blindern, Oslo, Norway
}

\begin{abstract}
During the last few years, quantum computing seems to have become a reality. Some few companies have developed quantum computers, and are in use in some large institutions and Universities, while other public organizations have made a similar attempt, but on a smaller scale. Promising to solve otherwise intractable problems for normal supercomputers, companies like Google are already using quantum computers to design solutions, including one allowing the software to distinguish cars from landmarks. However, after numerous and different empirical tests the results are not unanimous, resulting in new attempts to figure out what it is the best way of using this new type of machine that exhibits quantum properties. Developers and scholars argue for the need to redesign quantum computer to achieve full use of this new technology in computers. Proposing a designerly perspective on quantum computing seems a novel and interesting possibility to allow researchers fully taken advantages of this technology. This theoretical paper suggests the use of Design Thinking, and how it is used to solve wicked problems, to re-think how to approach quantum computing. For the design community, understand how to approach, understand and design for quantum computers, may open for a new field of research.
\end{abstract}

\section{KEYWORDS}

Quantum Computing, Design, Design Thinking, Wicked Problems

\section{A NEED FOR DESIGN IN QUANTUM COMPUTERS}

During the last few years, quantum computing seems to have become a reality. The Canadian company D-Wave (2019) has developed a quantum computer which is in use in few large institutions and Universities, while other public organizations have made similar attempts, but in smaller scale (Thompson 2014). The American company IBM has launched in 2017 an online service running on a quantum computer processor giving the possibility to the public to develop services (Lubensky 2017). In addition, both D-Wave and Berkeley based Rigetti (2019) has developed the same platform of services lately. The first commercial quantum super computer, the IBM Q System One, is also ready. Promising to solve otherwise intractable problems for normal supercomputers, companies like Google are already using a quantum computer to design solutions, including one allowing the software to distinguish cars from landmarks. On the other hand, after numerous and different empirical tests the results are not unanimous, resulting in new attempts to figure out, what it is the best way of using this new type of machine that exhibits quantum properties (Cusumano 2018; Grossman 2014). Quantum computers exploit proprieties of quantum mechanics laws, and, unfortunately, they are difficult to grasp as they show non-logical characteristics. As pointed by Coffey (2014) the need for a design approach is highly needed: "Physicists are making progress toward this goal every day, but nearly every part of a quantum computer still needs re-engineering or redesign to make it all work." Also, Google wrote in their research-blog a quest for new perspectives: "The design of next generation annealers must facilitate the embedding of problems of practical relevance." (Denchev et al. 2015; Google 2015) The idea of using a designerly way of thinking, to fully take advantages of this technology is novel. As an approach the paper uses Design Thinking (Brown 2009), as it has interesting elaborative forces aiming to define and solve problems. The design community has long traditions in approaching, analyzing and trying to solve complex problems (Dorst 2006), emphasizing on the creative designers activity between the problem space and solution space (ibid.) One category of problems of interest are those difficult or impossible to solve because of incomplete, contradictory, unbalanced and changing requirements (Rittel \& Webber 1973), they are named wicked. Using Design Thinking to solve ill-defined problems has proven as a viable way to develop solutions to them 
(Brown \& Wyatt 2010; Buchanan 1992; Jobst \& Meinel 2014; von Thienen, Meinel, \& Nicolai 2014). This theoretical paper suggests the use of Design Thinking, and how it is used to solve wicked problems, to re-think how to approach quantum computing.

\section{QUANTUM COMPUTING FOR DESIGNERS}

Quantum computing relays on theories of quantum mechanics, and unfortunately, they are difficult to grasp as they are often contradictory. They are non-logical. Quantum mechanics explain the rules working in the subatomic world. Thus, understanding quantum computing and the theories behind is not an easy task, and those limitations and constraints posed by technology need to be addressed properly. The design of the processor to be used in the quantum computer relays on the properties of some of the law of quantum mechanics. The first one, named superposition, is when, in the subatomic world, an electron acquires a state not positive or negative but in between, i.e. neither the one nor the other. In the world of computers, this corresponds to having a third-bit value in addition to 0 and 1 . When designing a processor, using the quantum bits named qubit, gives an exponential possibility of computation. The second property, entanglement, is when two entities, like electrons, after an interaction, becomes entangled, then a change in one entity will have an effect on the other one, regardless of distance. The last one is quantum decoherence of the state of superposition, where observation of the state in which the electron is resting down in one of the two possible positions. In quantum processor, the qubit decay from a fluid non-binary identity to one of the possible output. The superposition, entanglement, and quantum decoherence properties are needed to ensure maximal effectiveness in the quantum computer processor (Grossman 2014). In addition, there is a need to use an additional process so errors can be detected and corrected after decoherence.

\subsection{Superposition}

The most interesting propriety is superposition and implies a third position a particle can have in addition to a positive and negative state. This position is both negative and positive at the same time, but an uncertainty. The Schrödinger cat paradigm (Monroe, Meekhof, King, \& Wineland 1996) is an example often used to explain to non-scientist how superposition function. Inside a closed box, where the cat is, there is a radioactive item and a poison container. At some point, the radioactive item can activate and open the poison container with a beam. The observer outside cannot see if the beam has occurred, thus the cat is both alive and dead at the same time. For instance, in science, if a particle can have a spin left or right, a superposition implies that it has both simultaneously. Applied to computer science, a bit having the possibility to be in a superposition, can be represented by a quantum bit (qubits), and have as values 0 or 1 or both at the same time, representing a superposition. There are several types of processors using the laws of quantum mechanics, although they are using different hardware techniques (Ladd et al. 2010). The aforementioned D-Wave uses a niobium (a type of metal) based chip, where the spin of the atoms in one or the other direction represent the 0 or 1 used in normal computer processors. When elevated into a superposition, exponential quantity of data can be uploaded into the processor. This type of quantum computers uses "adiabatic quantum computing," also known as "quantum annealing." Common for all quantum processor is the problem with reading the state of the qubits (quantum decoherence), since it may happen uncontrolled by external temperature variation and noise, and therefore the processor need to be kept at the lower temperature possible and away of all form of noise.

\subsection{Entanglement}

The quantum property named entanglement implies a non-locality connection after an interaction has occurred between two particles (typically an electron). This connection acts counter-logical since it implies that each of the two particles are connected even if they are far away. Thus, changes made to one of the particles will affect simultaneously the other one. This fuzzy property act also when the two particles are moved far away from each other after the first interaction, leading to paradoxes (Rieffel 2000). Entanglement is often explained as a spooky action at a distance. Lately, tests have confirmed entanglement property of particles over large distances (Ursin et al. 2007). The implications of entanglement open for, among others, to quantum cryptography and quantum network (Sangouard, Simon, de Riedmatten, \& Gisin 2011). 


\subsection{Quantum Decoherence}

Decoherence is a quantum behavior happening when a particle (typically an electron) in superposition do rest down to a classical state, as a reaction to an interaction, like observation or noise. In a qubits decoherence results in moving from a state of quantum superposition back to one of the two states e.g. 0 or 1 . If not controlled properly, the effect of decoherence may cause insecurity in regards to the validity of the answer, since the way the observation is done will reflect on the result.

\subsection{How a Quantum Computer Works}

Summing up, the quantum computer uses processor where "entanglement makes it possible for quantum bits to work together and represent multiple combinations of values simultaneously, rather than represent one combination at a time." (Cusumano 2018) Qubits, in addition to being entangled, when in a superposition state, they can store and represent an exponential quantity of data. The aforementioned niobium processor supports this feature. The combination of representing and storing an exponential amount of values make the quantum computer extraordinary powerful. The values represented can be a complete set of solutions or answers to a given problem. The clue of the niobium processor is the ability to analyze all the possible answers at the same time, and at the end, the answer with the highest cumulative probability will be the correct answer (Trabesinger 2017). Rephrased, when decoherence is performed on all the qubits of a quantum processor the correct answer will be discovered among all answers. However, several iterations of the same process are needed to achieve a relevant cumulative probability.

\section{DESIGN THINKING AND QUANTUM COMPUTING}

Design Thinking uses a combination of immersive understanding of context and users and their needs in the problem space. The rapid prototyping act during a Design Thinking process fosters the best possible solution. Abductive reasoning (Brown 2009; Kolko 2012) is one of the elaborative force behind Design Thinking, which helps define the solution space. This way of thinking helps redefine the problem while finding a solution. The elaborative force behind this activity support a converge toward to a solution and in doing so, a redefinition of the problem (Kolko 2009).

The rapid prototyping act in the Design Thinking process, rely upon a divergent thinking act (Cropley 2006), adding insight in the process where the outcome is a high quantity of results and prototypes. This activity of making alternative solutions is mediated by "a variety of tensions between possibilities and constraints." (Liedtka 2015) Convergent thinking is then needed to support an abductive reasoning process narrowing the results, and giving at the end a handful of viable ideas. Rowe (Rowe 1987) says also that the action (of designing) and the problem definition becomes intertwined, as the problem become defined simultaneously with the proposal of a solution. Overall, the process of Design Thinking, allows the result to be a technically viable solution, a desirable output for users and an economically feasible project (Brown 2009).

Another strength Design Thinking has, is the possibility to solve complex and ill-defined problems (Brown \& Wyatt 2010; Rittel \& Webber 1973), ranging from business to social context. Usually problems in science have a single goal and predefined rules enabling a clear solution (Coyne 2005); they can be defined as tame. Mathematics is one example, where solving an equation require little creative effort as one need to follow a clear predefined rule in how to solve them. On the contrary, wicked problems belongs to the architectural, social and organizational sphere. Wicked problems are complex, ill-defined and are hardly to fully be solved. Those problems have no stopping rules, and they are always changing and evolving, driven by a variety of stakeholders (Churchman 1967; Rittel \& Webber 1973). Rittel has identified ten properties of wicked problem, where two are relevant in regards to quantum computing: solutions to wicked problems "are not true-or-false, but good-or-bad", and every solution to a wicked problem is a "one-shot operation". In the next section, a discussion will clarify how to apply the designedly approach used to solve wicked problems, to exploit the possibility quantum mechanics give when developing a quantum computer. 


\section{DISCUSSION}

Wicked problems show signs of good analogies with quantum computing, especially with superposition, entanglement, and decoherence. As a possible solution to wicked problems, Buchanan (1992) use the "concept of placements" which bring to the design process insight and different perspectives. Constructing a design situation with different "placements", the problem formulation and the solution coincide (Johansson-Sköldberg, Woodilla, \& Çetinkaya 2013).

In Design Thinking, the problem formulation and the solution are described as the result of abductive reasoning. Abduction in reasoning was first described by Pierce as the "step of adopting a hypothesis as being suggested by the facts" (Pierce 1974). One of the relevant points from a design perspective is the fact that abductive reasoning function also as a generator of new knowledge and insight (Fischer 2001; Kolko 2009). The solution is not part of the premises, but as a result of an educated guess, based on observations, data, and insight (Kolko 2009). In other words, the problem becomes re-defined at the same time as the proposed solution (Khisty 2000; Rowe 1987). New understandings are created. This framework relates with Buchanan "placements", and explains why it is possible to argue that Design Thinking can solve wicked problems.

As mentioned the niobium processor can analyze all the possible answers of a problem at the same time (Trabesinger 2017). However, applying how Design Thinking can solve wicked problems, can inform how to exploit the full potential of the quantum processor. During the process of redefining the problem, new data are created (e.g. knowledge) and are needed to be used in the same process. The design of the quantum processor should take this into account.

\section{CONCLUSION AND FUTURE WORK}

The field of quantum computing is still at the first steps toward a promising new future, as researchers are still trying to figure out what it's in for them and how to use it properly. The analogy between how the Design Thinking approach can be used to solve wicked problems, and the different quantum mechanics properties presented in this paper, gives a new understanding in how to address and adapt design methods to quantum computing. Future work should analyze other types of quantum processor and how Design Thinking can help a redesign.

\section{REFERENCES}

Brown, T 2009, Change by design: how design thinking transforms organizations and inspires innovation, HarperCollins Publishers, New York.

Brown, T \& Wyatt, J 2010, 'Design Thinking for Social Innovation (SSIR)' Stanford Social Innovation Review, vol. 30, no. 5 .

Buchanan, R 1992, 'Wicked Problems in Design Thinking' Design Issues, vol. 8, no. 2, pp. 5-21, doi: 10.2307/1511637.

Churchman, CW 1967, 'Guest Editorial: Wicked Problems' Management Science, vol. 14, no. 4, pp. B141-B142.

Coffey, VC 2014, 'The Incremental Quest for Quantum Computing' PHOTONICS SPECTRA, vol. 48, no. 6, pp. 36-41. Coyne, R 2005, 'Wicked problems revisited' Design Studies, vol. 26, no. 1, pp. 5-17, doi: 10.1016/j.destud.2004.06.005. Cropley, A 2006, 'In Praise of Convergent Thinking' Creativity Research Journal, vol. 18, no. 3, pp. 391-404.

Cusumano, MA 2018, 'The Business of Quantum Computing' Communications of the ACM, vol. 61, no. 10, pp. $20-22$.

Denchev, VS, Boixo, S, Isakov, SV, Ding, N, Babbush, R, Smelyanskiy, V, Martinis, J, \& Neven, H 2015, 'What is the Computational Value of Finite Range Tunneling?' ArXiv:1512.02206 [Quant-Ph]

Dorst, K 2006, 'Design Problems and Design Paradoxes' Design Issues, vol. 22, no. 3, pp. 4-17.

D-Wave 2019, 'D-Wave Systems', viewed 10 October 2018, http:/www.dwavesys.com/.

Fischer, HR 2001, ‘Abductive Reasoning as a Way of Worldmaking' Foundations of Science, vol. 6, no. 4, pp. 361-383,

Google 2015, 'When can Quantum Annealing win?', Retrieved from http://googleresearch.blogspot.com/2015/12/whencan-quantum-annealing-win.html.

Grossman, L 2014, February 6, 'The Quantum Quest for a Revolutionary Computer' TIME, Retrieved from http://time.com/4802/quantum-leap/. 
International Conferences Interfaces and Human Computer Interaction 2019; Game and Entertainment Technologies 2019; and Computer Graphics, Visualization, Computer Vision and Image Processing 2019

Jobst, B \& Meinel, C 2014, 'How Prototyping Helps to Solve Wicked Problems' in L. Leifer, H. Plattner, \& C. Meinel (eds.), Design Thinking Research: Building Innovation Eco-Systems, pp. 105-113, Springer International Publishing, Cham, doi: 10.1007/978-3-319-01303-9_8.

Johansson-Sköldberg, U, Woodilla, J, \& Çetinkaya, M 2013, 'Design Thinking: Past, Present and Possible Futures' Creativity and Innovation Management, vol. 22, no. 2, pp. 121-146, doi: 10.1111/caim.12023.

Khisty, C 2000, 'Can Wicked Problems Be Tackled through Abductive Inferencing?' Journal of Urban Planning and Development, vol. 126, no. 3, pp. 104-118, doi: 10.1061/(ASCE)0733-9488(2000)126:3(104).

Kolko, J 2009, 'Abductive Thinking and Sensemaking: The Drivers of Design Synthesis' Design Issues, vol. 26, no. 1, pp. 15-28, doi: 10.1162/desi.2010.26.1.15.

Kolko, J 2012, Wicked problems: problems worth solving: a handbook and call to action, Ac4d, Austin Center for Design, Austin, Texas.

Ladd, TD, Jelezko, F, Laflamme, R, Nakamura, Y, Monroe, C, \& O’Brien, JL 2010, 'Quantum computers' Nature, vol. 464, no. 7285, pp. 45-53, doi: 10.1038/nature08812.

Liedtka, J 2015, 'Perspective: Linking Design Thinking with Innovation Outcomes through Cognitive Bias Reduction' Journal of Product Innovation Management, vol. 32, no. 6, pp. 925-938, doi: 10.1111/jpim.12163.

Lubensky, D 2017, March 6, 'Quantum computing gets an API and SDK', Retrieved from https://developer.ibm.com/dwblog/2017/quantum-computing-api-sdk-david-lubensky/.

Martin, R 2009, The design of business: why design thinking is the next competitive advantage, Harvard Business School Press, Boston.

Monroe, C, Meekhof, DM, King, BE, \& Wineland, DJ 1996, 'A "Schrödinger Cat" Superposition State of an Atom' Science, vol. 272, no. 5265, pp. 1131-1136, doi: 10.1126/science.272.5265.1131.

Pierce, CS 1974, Collected Papers of Charles Sanders Peirce ed. C. Hartshorne, Harvard University Press, Boston, Mass., Retrieved from http://www.hup.harvard.edu/catalog.php?isbn=9780674138001.

Rieffel, E 2000, 'An Introduction to Quantum Computing for Non-physicists' ACM Comput. Surv., vol. 32, no. 3, pp. 300-335, doi: 10.1145/367701.367709.

Rigetti 2019, 'Rigetti Computing', Rigetti Computing, viewed 30 May 2019, https://www.rigetti.com/news.

Rittel, HWJ \& Webber, MM 1973, 'Dilemmas in a general theory of planning' Policy Science, vol. 4, pp. 155-169.

Rowe, PG 1987, Design thinking, MIT Press, Cambridge, Mass.

Sangouard, N, Simon, C, de Riedmatten, H, \& Gisin, N 2011, 'Quantum repeaters based on atomic ensembles and linear optics’ Reviews of Modern Physics, vol. 83, no. 1, pp. 33-80, doi: 10.1103/RevModPhys.83.33.

Stewart, J, Gold, J, Gold, J, Walton, J, Cureton, P, \& Anderson, L 2011, 'Theorising and practitioners in HRD: the role of abductive reasoning' Journal of European Industrial Training, vol. 35, no. 3, pp. 230-246

Thompson, C 2014, May 20, 'The Revolutionary Quantum Computer That May Not Be Quantum at All' WIRED, Retrieved from http://www.wired.com/2014/05/quantum-computing.

Trabesinger, A 2017, 'Quantum leaps, bit by bit.' Nature, vol. 543, no. 7646, pp. S2-S3, doi: 10.1038/543S2a.

Ursin, R, Tiefenbacher, F, Schmitt-Manderbach, T, Weier, H, Scheidl, T, Lindenthal, M, Blauensteiner, B, Jennewein, T, Perdigues, J, Trojek, P, Ömer, B, Fürst, M, Meyenburg, M, Rarity, J, Sodnik, Z, Barbieri, C, Weinfurter, H, \& Zeilinger, A 2007, 'Entanglement-based quantum communication over 144 km' Nature Physics, vol. 3, no. 7, pp. 481-486, doi: 10.1038/nphys629.

von Thienen, J, Meinel, C, \& Nicolai, C 2014, 'How Design Thinking Tools Help To Solve Wicked Problems' in L. Leifer, H. Plattner, \& C. Meinel (eds.), Design Thinking Research: Building Innovation Eco-Systems, pp. 97-102, Springer International Publishing, Cham, doi: 10.1007/978-3-319-01303-9_7. 